\title{
SSNN-Based Energy Management Strategy in Grid Connected System for Load Scheduling and Load Sharing
}

\author{
Yuvaraja Teekaraman ${ }^{1 D},{ }^{1}$ K. A. Ramesh Kumar, ${ }^{1}$ Ramya Kuppusamy, ${ }^{1}$ \\ and Amruth Ramesh Thelkar $\mathbb{( D}^{2}$ \\ ${ }^{1}$ Department of Energy Science (Formerly Energy Studies), Periyar University, Salem City 636011, India \\ ${ }^{2}$ Faculty of Electrical \& Computer Engineering, Jimma Institute of Technology, Jimma University, Jimma, Ethiopia \\ Correspondence should be addressed to Yuvaraja Teekaraman; yuvarajastr@gmail.com and Amruth Ramesh Thelkar; \\ amruth.rt@gmail.com
}

Received 25 October 2021; Revised 22 November 2021; Accepted 10 December 2021; Published 10 January 2022

Academic Editor: Ravi Samikannu

Copyright (C) 2022 Yuvaraja Teekaraman et al. This is an open access article distributed under the Creative Commons Attribution License, which permits unrestricted use, distribution, and reproduction in any medium, provided the original work is properly cited.

\begin{abstract}
The proposed research work focused on energy management strategy (EMS) in a grid connected system working in islanding mode with the connected renewable energy resources and battery storage system. The energy management strategy developed provides a balancing operation at its output by utilizing perfect load sharing strategy. The EMS technique using smart superficial neural network (SSNN) is simulated, and numerical analyses are presented to validate the effectiveness of the centralized energy management strategy in a grid connected islanded system. A SSNN prediction model is unified to forecast the associated household load demand, PV generation system under various time horizons (including the disaster condition), EV availability, and status on EV section and distance. SSNN is one the most reliable forecasting methods in many of the applications. The developed system is also accounted for degradation battery model and its associated cost. The incorporation of energy management strategy (EMS) reduces the amount of energy drawn from the grid connected system when compared with the other optimized systems.
\end{abstract}

\section{Introduction}

Energy storage and management play a vital role in the installation of the smart grid system as they increase the stability, resiliency, and efficiency of the grid connected system. In today's scenario, generating power using renewable energy resources needs to be balanced dynamically with the dynamic changing load. The smart grid operator has limited resources and techniques to maintain stability in the present changing atmospheric condition and load profiles.

The power conversion system plays a prominent role in the grid connected renewable energy resource system. The most challenging phenomenon in the power distribution system is the loss occurring in the conversion system. The power conversion system is utilized when the power is required to transfer it from source to load; else the process manages to store the energy in the connected storage system [1]. Genetic algorithm is used for better system optimization, and it has gained importance in reducing the cost of electricity [1]. In integrating the energy storage system with the power generation system, an effective operating point on storage capacity needs to be considered. Reinforcement learning is incorporated in battery sizing and real-time EMS [2].

Electric traction is the most prominent area for implementing the energy storage and energy management technique.

Model predictive control algorithm was utilized in railway control activity to improve the system efficiency and to reduce the operating cost of the system [3]. The integration of RES like solar, wind, geothermal, and biomass with the grid addresses many issues towards environmental pollution and energy scarcity [4]. Decentralized control strategy architecture is used for better energy management. The uncertainties in the RES connected system [5] are handled by modeling the EMS at the level of individual grid 
and in turn can be configured for multistage systems. Energy storage in the grid connected system provides larger benefits. The modeling of BESS along with BMS in [6] is categorized based on time scale. The study made by the authors in [6] investigates the benefits of energy storage during power generation, distribution, and power transmission. Due to energy crisis, RES is integrated with the present conventional sources system.

The integrated grid allows the customer to use the load. The HEMS installed at home server collects all the data pertaining to load usage, analyzes the collected data, and develops the control strategy for reducing the cost of electricity [7]. The control strategy in [8] compares the grid connected system with centralized and decentralized control strategies. Implementing the CCS and DCS provides output comparative statement in terms of battery life cycle, cost of electric energy, and battery size determination. Existing energy management techniques discussed in [9] examine the effect of system level energy reduction strategy in accordance with the energy consumption of RTS which uses duplication for fault tolerance. The comparative analysis gives a key note to the researcher for deciding the most appropriate energy storage and management strategy for particular application. The energy management strategies installed in the grid connected system [10] ensure complete system stability and economic level of operation. The multiagent HEMS (MAHEMS) includes the feature of both CCS and DCS. Therefore, the MAHEMS is developed as 3 levels of EMS framework that provides both local and autonomous level of coordinated energy control in centralized grid operation. With the development of smart grid, energy transition happens from passive energy consumers to an active smart grid supplier [11]. The integration of RES in [12] plays a vital role in future energy system. The use of RES as integrated resources reduces $\mathrm{CO}_{2}$ emission with the high-level penetration of RES in various transmission system operators. Grid with an integrated RES with energy storage and curtailable load and noncurtailable load provides low-voltage distribution system (LVDS). Smart grid is a novel approach that clusters the small distributed generators. The variable power generation, load profiles, and energy cluster parameters are compared with decision tree approach under different battery energy storage conditions. The universal energy storage and management strategy in [13] produce result as reduction in energy saving as compared with the traditional approach. The present available PHEV in [14] is being connected with battery as single energy storage system. The battery has limited power density due to its inherent chemical characteristics, which affects the performance of the PHEV system. The objective focused in [14] minimizes the usage of electricity in EV and increases the lifetime of the battery. The control strategy as two-level structure for the integrated hybrid standalone system is discussed in [15]. Due to continuous change in atmosphere, the uncertainties occurring in RES are handled by the two-level control strategy and provide perfect energy management, power regulation, and load scheduling operation and control.

\section{System Architecture}

The grid connected system can be operated either by centralized control strategy (CCS) or by means of decentralized control strategy (DCS). In the case of decentralized control strategy (DCS), individual customer pertaining to the load usage will deal all sort of energy transactions directly with the grid, whereas it is not in the case of centralized control strategy (CCS). The fishbone diagram of centralized control strategy is shown in Figure 1. The centralized strategy shown in Figure 1 is the aggregator which is responsible to make energy transactions between the grid and various loads (EV system, household loads, and industrial loads). The grid support services are provided by the individual customer in DCS and the aggregator in CCS. The fishbone shown in Figure 1 acts as an aggregator which groups all the agents connected in a power system and acts as a single object in energy markets/grid operator services. The fishbone as aggregator performs demand response operation, monitors various connected loads, and controls system equipment, energy generation, and storage sources. The developed fishbone model for CCS reduces the electric tariff for an individual customer and shared stakeholders and prepares an augmented schedule for charging/discharging of EV and connected stationary battery based on the most prominent time of power generation by the PV system. The fishbone connected to the CCS completely owns and manages the connected PV source, fuel cell power module, and the battery connected system.

The EV system owners send their augmented schedule preferences for charging/discharging of EV to the aggregator. The fishbone as aggregator is a place for predicting the power required to handle the connected load profiles based on the weather condition. The weather condition and the time horizon (short term, midterm, and long term) develop a series of dataset in solar PV generation. The status of the system components under various time horizons is shown in Table 1. Based on this historical time series datasets, the aggregator is having the access to towards the connected loads, EV charging and discharging station, connected PV source, grid connected power station, and the battery storage system.

\section{Energy Management Proposed Flow Model}

The energy management strategy's process flow model comprises the (i) optimization model, (ii) prediction model, (iii) battery operation model, and (iv) validation model. The function hold by each of the process flow model is represented in Figure 2. The proposed EM strategy bifurcates its operation in terms of load sharing and managing by scheduling the process of charging/discharging in daily basis and governs the energy supply and demand to reduce the tariff rate. The proposed EMS model analyzes the connected load, connected PV resources, and required power for EV charging/discharging station based on time horizon. The fishbone aggregator model analyzes all the required loads that are connected to the interconnected system and communicates the charging/discharging schedule of EV 


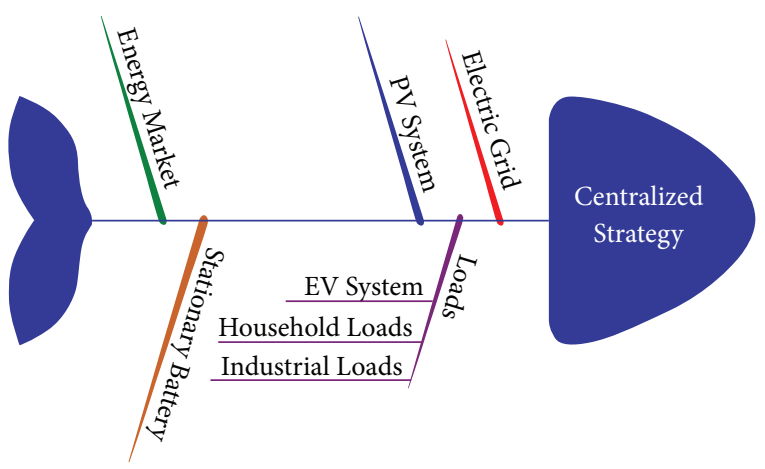

FIGURE 1: Fishbone aggregator architecture diagram of centralized strategy.

based on the mixed-integer linear programming (MILP) model subjected to grid constraints. The load predictions for the day are predicted and given as input to optimization model in order to formulate the optimum schedule of charging/discharging required for the EV station and other connected battery storage systems.

$$
\mathscr{F}_{t}^{\text {loads }}=\sum_{j=1}^{n} \boldsymbol{\Omega}_{j} f\left[\mathscr{W}_{0 j}+\sum_{i=1}^{x} \mathscr{W}_{i j} \mathscr{F}_{t-1}^{\text {loads }}\right]+\mathfrak{R}_{t}+\mathfrak{\Omega}_{0}
$$

\section{Energy Prediction Model}

A smart superficial neural network (SSNN) prediction model is incorporated to predict the connected household load demand, PV generation system from different time horizons, EV availability, and status on EV section and distance. SSNN is one the most reliable forecasting methods in many of the applications. A commonly used backpropagation algorithm (BPA) is utilized in feedforward mode. The forecasted value in terms of connected household load demand and PV generation system from different time horizons can be expressed as follows:

where equation (1) refers to the forecasted value of the demand with reference to connected load and the power availability in PV generation system. The forecasted value is calculated using SSNN, in which the weight function and the bias variable decide the amount of load to be handled in the connected system.

$\mathscr{F}_{\mathrm{t}}$ loads-Forecasted value in terms of connected household load demand and PV generation system, $\boldsymbol{\Omega}_{\mathrm{j}}$ and $\mathscr{W}_{\mathrm{ij}^{-}}$ Weights from the layer, $n$-Number of hidden layers in SSNN model, $\boldsymbol{\Omega}_{0}$ and $\mathscr{W}_{0 \mathrm{j}}$-Bias terms of SSNN model, Loads $\in \mathscr{L}_{\mathrm{t}}$
$\mathscr{K}_{\mathrm{t}}-\mathscr{L}_{\mathrm{t}}$ : household loads and $\mathscr{K}_{\mathrm{t}}:$ PV generation system, tTime.

The next parameter in the prediction model is EV availability. The existing antique time series data for EV travel pattern are utilized to extract probability distribution function (PDF) for categorizing the EV availability with reference to travel distance and range efficiencies. The EV availability for charging and discharging is represented in terms of binary definition as " 0 " when EV is "not available" and "1" when EV is "available." The EV availability as a function of time " $t$ " and day " $d$ " of the week is expressed as in equation (2) subjected to varying atmospheric temperature. The flow diagram showing the EV availability status is shown in Figure 3.

$\mathbb{E} \mathbb{V}_{t}=f\left(t \| \mathbb{S}_{E V, t}, \mathbb{P}_{E V, t}\right)=\left(\begin{array}{c}\mathbb{S}_{E V, t} \\ \mathbb{P}_{E V, t}\end{array}\right) \mathbb{P}_{E V, t}\left(1-\mathbb{P}_{E V, t}\right)^{\left(\mathbb{S}_{E V, t}-t\right)}$,

where $\mathbb{E} \mathbb{V}_{t}$ represents the EV availability w.r.t time, $\mathbb{S}_{E V, t}$ represents the number of scenarios available in EV w.r.t time, and $\mathbb{P}_{E V, t}$ represents the PDF of EV scenarios.

The algorithm to find the EV availability status is shown below. The value given in the $\mathrm{EV}$ availability status algorithm is calculated as a function of time, day of the week at varying temperature as given in equation (2). At $t=12$ noon, the EV availability is measured in terms of insolation. The available insolation at 12 noon is 1000 . From $t=8$ am to $t<12$ noon, the insolation measured is 650 , and it is applied in equation (2), and the obtained EV status is 3 and 2, depending on the variation occurring due to atmospheric condition. For $t>12$ noon, the insolation measured varies from 700 to 990 and the obtained EV status is 4 and 2 (Algorithm 1).

\section{Battery Operation Mathematical Modeling}

The state of charge (SOC) and the state of health ( $\mathrm{SOH}$ ) define the battery life. The SOC on EV at any time $t$ is modeled by using equation (3) based on SOC on EV at time $t=t-1$, charging/discharging efficiency on EV battery, charging/discharging power on $\mathrm{EV}$, and maximum $\mathrm{EV}$ battery capacity. By specification, each battery has its own maximum and minimum SOC. Based on the specification according to equation (4), the modeled system considers its upper and lower limits of battery SOC and its distance travelled when EV is away from the substation so as to improve the effectiveness of battery $\mathrm{SOH}$.

$$
\mathrm{SOC}_{t}^{\mathrm{EV}}=\mathrm{SOC}_{t-1}^{\mathrm{EV}}+\frac{\eta_{\mathrm{EVC}} \mathbb{P}_{\mathrm{EVC}, t} \mathbb{A}_{t} \mathscr{F} t}{\mathbb{A U I}_{E V, \text { max }}}-\frac{\mathbb{P}_{E V d, t} \mathbb{A}_{t} \mathscr{F} t}{\eta_{E V d} \mathrm{Al}_{E V, \text { max }}}, \quad \leftrightarrow \mathbb{A}_{t}=1, t \in T,
$$

where $S O C_{t}^{E V}$ represents the state of charge on EV battery at time " $t$," $\eta_{E V C}$ represents the charging efficiency on EV battery, $\eta_{E V d}$ represents the discharging efficiency on EV battery, $S O C_{t-1}^{E V}$ represents the state of charge on EV battery at time " $t-1$, , $\mathbb{P}_{E V C, t}$ represents the maximum EV battery charging power $(\mathrm{kW}), \mathbb{P}_{E V d, t}$ represents the maximum EV battery discharging power $(\mathrm{kW}), \mathbb{A}_{t}$ represents the availability matrix, $\mathbb{A} \mathbb{H}_{E V, \text { max }}$ represents the maximum battery 
TABLE 1: Connected system status under various time horizons.

\begin{tabular}{lcccccc}
\hline Scenario & Status of PV system & $\begin{array}{c}\text { Stationary battery } \\
\text { usage }\end{array}$ & $\begin{array}{c}\text { Gas turbine } \\
\text { system }\end{array}$ & $\begin{array}{c}\text { Thermal } \\
\text { storage system }\end{array}$ & Cost minimization & $\mathrm{Maximum}_{2} \mathrm{CO}_{2}$ emissions \\
\hline $\begin{array}{l}\text { Short-term } \\
\text { horizon }\end{array}$ & No & No & No & No & Yes & No \\
$\begin{array}{l}\text { Midterm } \\
\text { horizon }\end{array}$ & Yes & Yes & Yes & No & Yes & No \\
$\begin{array}{l}\text { Long-term } \\
\text { horizon }\end{array}$ & Yes & Yes (discounted) & Yes & Yes & Yes & No \\
\hline
\end{tabular}

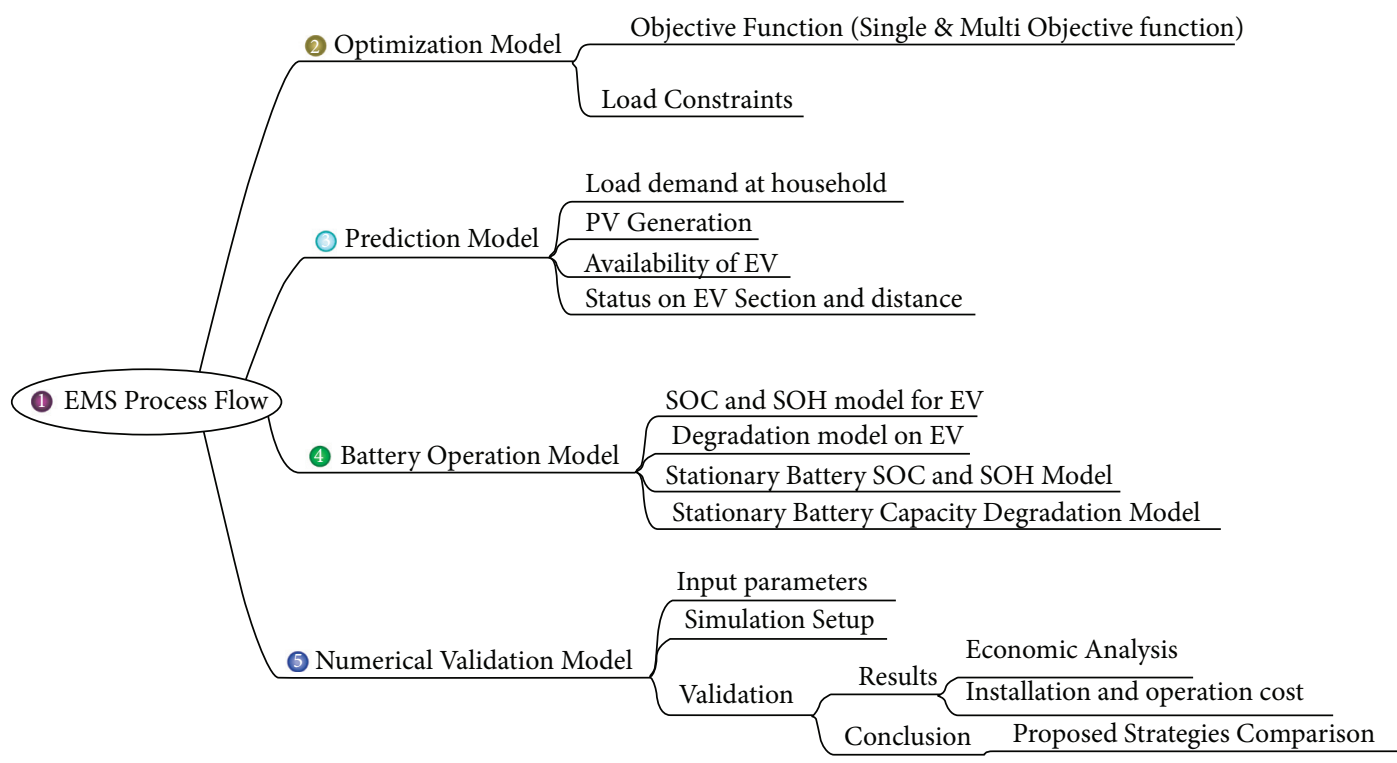

Figure 2: Energy management strategy process flow.

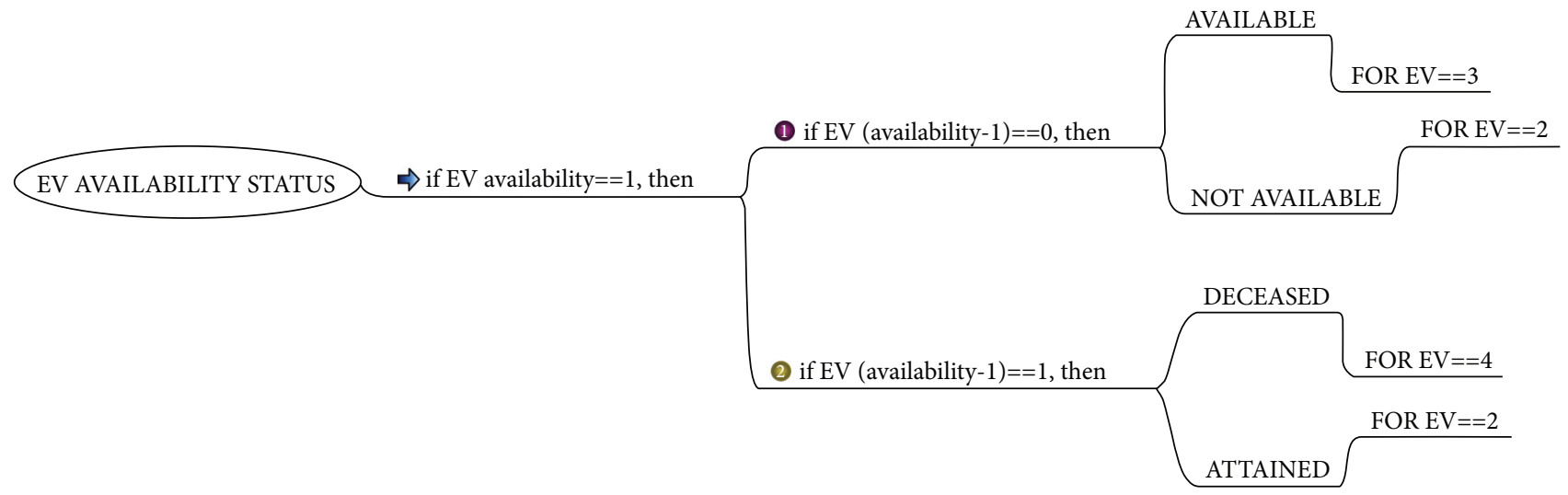

FIgURE 3: Flow diagram of EV availability status.

capacity in ampere hour, $\eta_{E V}$ represents the efficiency on EV battery $(\mathrm{KWh} / \mathrm{km})$, and $d_{E V}$ represents the distance travelled by $\mathrm{EV}$.

$$
\mathrm{SOC}_{t}^{E V}=\mathrm{SOC}_{t-1}^{E V}-\frac{\eta_{E V} d_{E V}}{\mathbb{A} \mathbb{W}_{E V, \text { max }}}, \quad \leftrightarrow \mathbb{A}_{t}=0, t \in T
$$

The degradation phenomenon in EV battery is observed in rechargeable batteries that reduce the lifetime of the battery over the specific period of time. This in turn increases the operating cost. Therefore, while modeling the battery degradation model, cost should be taken into account along with all the services.

\section{Optimization Model}

The main objective of developing the optimization model is to minimize the electric tariff. The process flow optimization model is shown in Figure 4, and the objective function is 


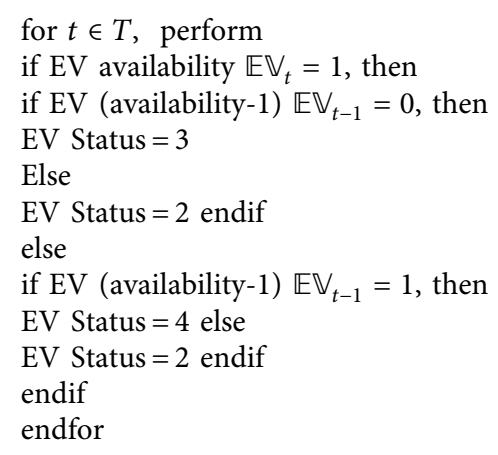

Algorithm 1: EV availability status algorithm.

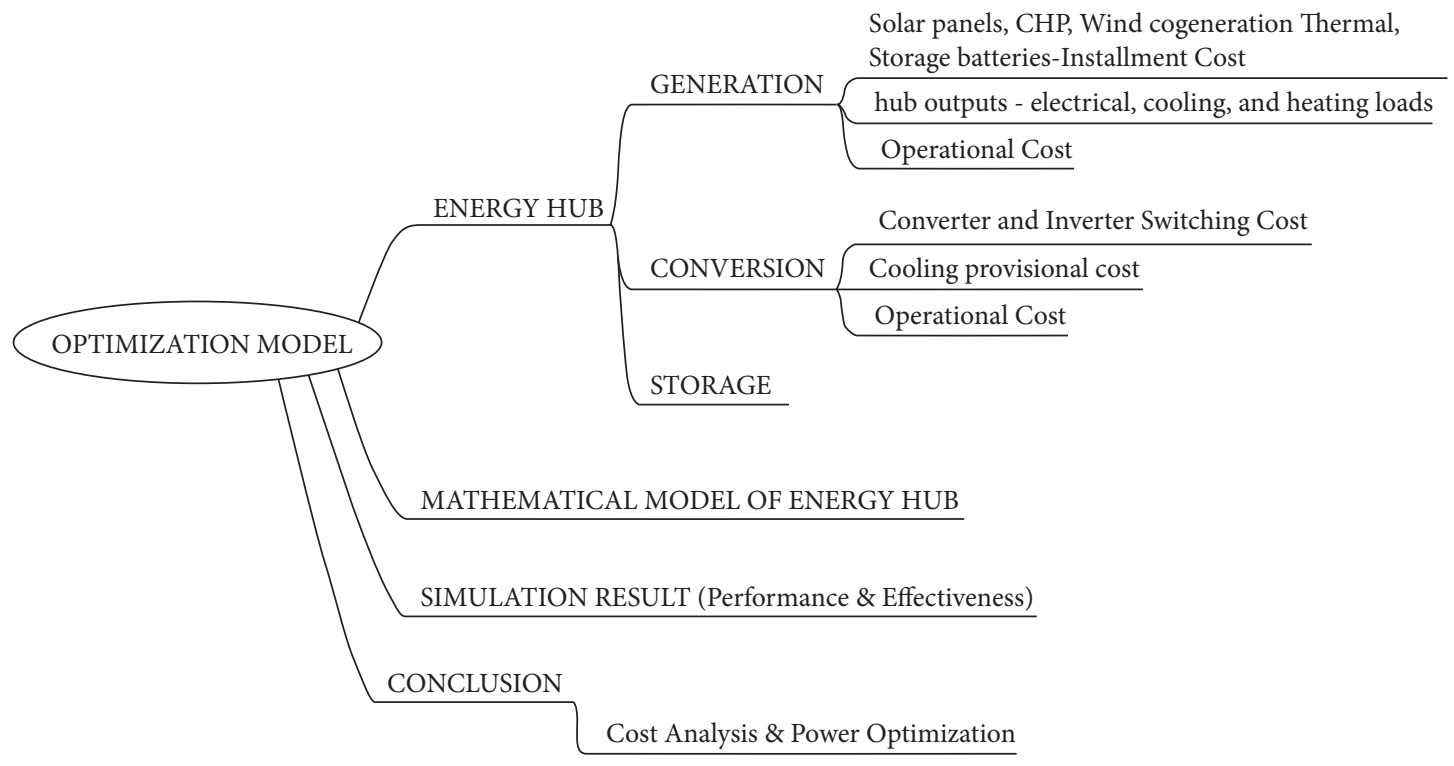

Figure 4: Process flow optimization model.

developed based on energy purchased, energy sold, and the degradation cost.

The expected operating cost of the asset is expressed as follows:

$\mathrm{OC}=$ energy purchased + energy sold + degradation cost,

$O C=\sum_{t \in T}\left(\alpha P_{t} \Delta t\right) \$_{p}+\left(\beta P_{t} \Delta t\right) \$_{S}+\left(\$_{d}^{E V}+\$_{d}^{B}\right)$,

where $P_{t}$ is the power flow to and from grid at any time " $t, " \alpha$ and $\beta$ are binary variables for power drawn and power supplied to or from the grid, $\$_{p}$ is the purchased energy tariff, and $\$_{S}$ is the energy sold tariff. Table 2 presents the binary variable constraints and their status. Energy is purchased to the grid, when $\alpha=1$ and $\beta=0$; under this condition, the power drawn from the grid is positive. Energy is sold from the grid, when $\alpha=0$ and $\beta=1$; under this condition, the power drawn from the grid is negative.
TABLe 2: Binary variable constraints and their status.

\begin{tabular}{llll}
\hline & \multicolumn{1}{c}{ PCC status } & $\alpha$ & $\beta$ \\
\hline Energy purchase & Power drawn from the grid is positive & 1 & 0 \\
Energy sold & Power drawn from the grid is negative & 0 & 1 \\
\hline
\end{tabular}

\section{Simulation Setup and Test Results}

The proposed strategy is developed for the given system configuration as shown in Table 3, using MATLAB, and its simulation set of energy management system with hybrid sources is as shown in Figure 5. The PV source is connected to the grid connected system PCC point via the energy storage systems like fuel cell, battery, and supercapacitor. The system is operated at discrete time interval with an operating switching frequency of $10 \mathrm{KHz}$. The energy management system (EMS) controls the power of the entire energy source devices through reference signals. 
TABLE 3: System configuration.

\begin{tabular}{lc}
\hline Parameter & System rating \\
\hline Solar panel & Polycrystalline solar panel \\
Number of solar panels connected & 70 nos. \\
Total number of stationary battery units & 10 nos. \\
Battery AH & $5 \mathrm{AH}$ \\
Household load capacity & $6 \mathrm{~kW}$ \\
\hline
\end{tabular}

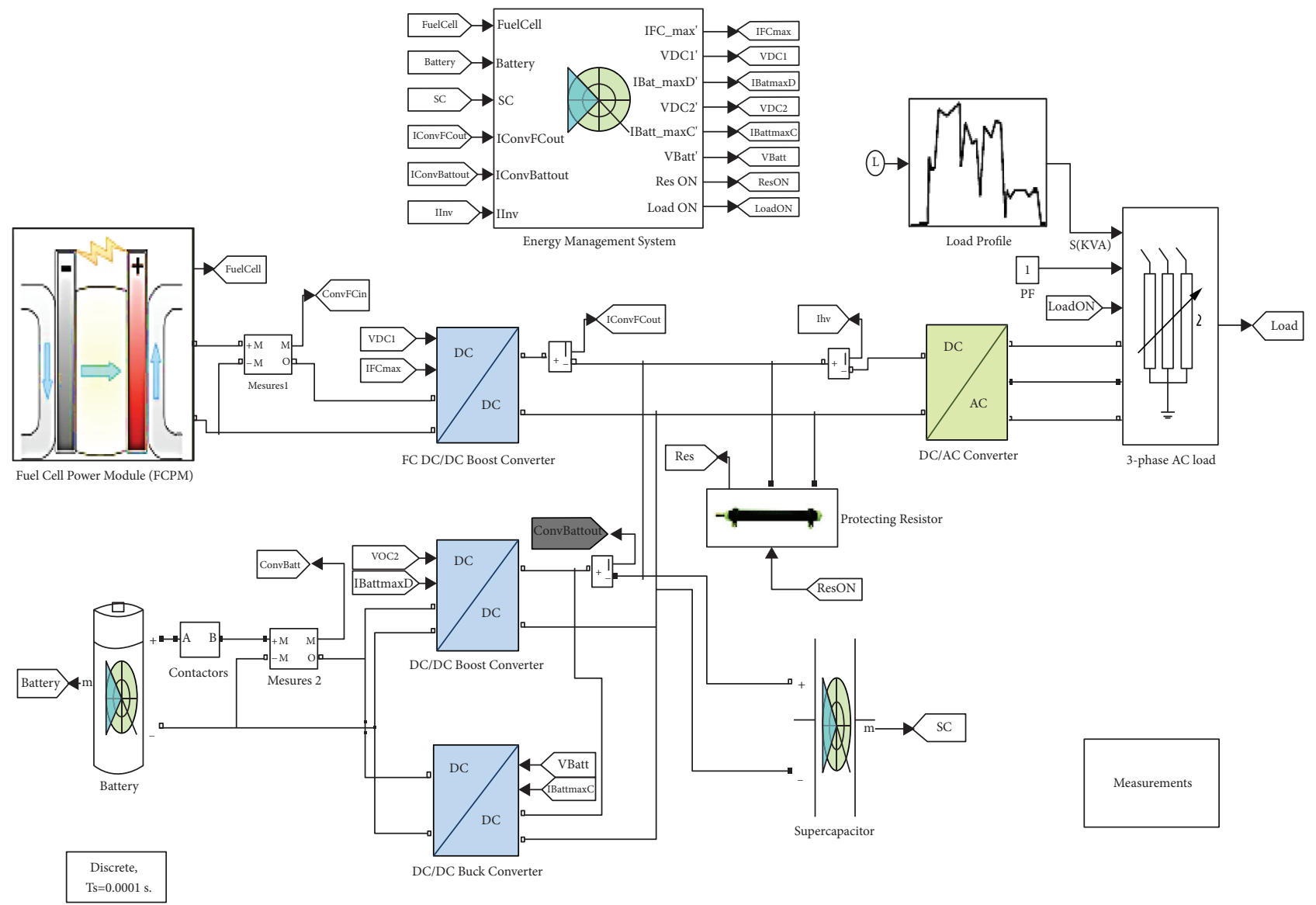

FIGURE 5: Simulation of grid connected system with its energy storage.

The subsystem of energy system modeling is shown in Figure 6 which provides the state machine control strategy. The simulation of the grid connected islanding mode network uses the ANN prediction model to generate the household load and the details of solar PV generation. The nntool in MATLAB is utilized to develop feed-forward backpropagation algorithm. It comprises the input layer (time of the day), hidden layer (load schedule), output layer (power gets distributed to the load according to the required schedule). The hidden layer neuron is assigned with the membership function of tansig (hyperbolic tangential sigmoidal), and the output layer is assigned with purelin (pure linear function) membership function. The SSNN showing the details of the attributes towards the input layer, hidden layer, and output layer is shown in Figure 6. The prediction model developed uses the three-layer network and is trained by the Levenberg-Marquardt algorithm (LMA). LMA is one of the most prominent algorithms as it is operative in categorizing the least convex objective function. The integration of the steepest descent method and the Gauss-Newton quadratic convergence method makes it possible in categorizing the least convex objective function. Increasing the power availability in the system makes perfect system load forecast schedule.

Load scheduling is done based on power generation in the integrated grid system. Figure 7 shows the PV system inclination angle analysis using fuzzy ANN. As shown in Figure 7, the amount of solar irradiance falling on the solar PV system is increased when the tilt angle is at $10.1^{\circ}$. During this period of time, the amount of power generation is more. Based on this sample, the PV dataset is taken and analyzed as shown in Figure 8.

Since the irradiance is not constant throughout the day, power generation will not be constant. To supply constant 


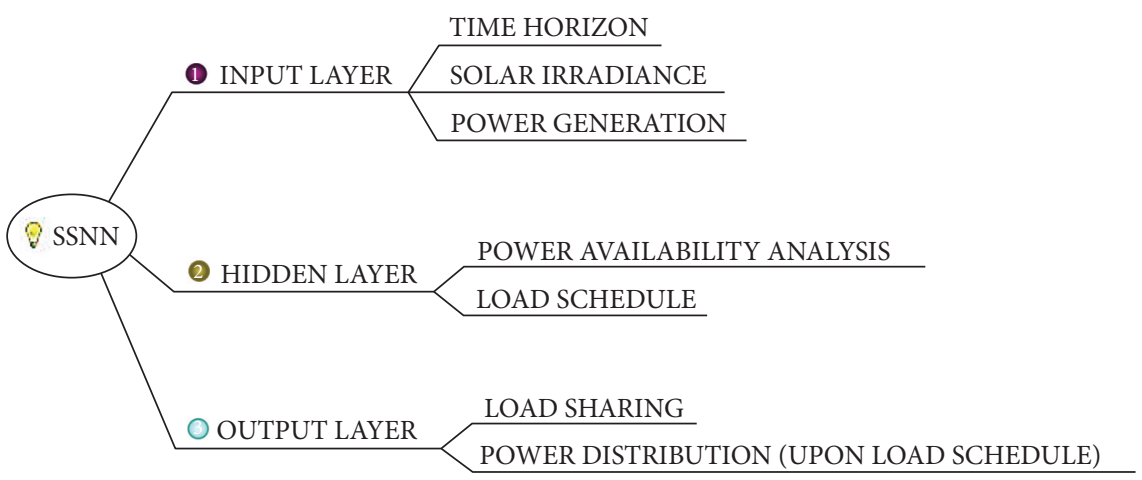

FIGURE 6: Smart superficial neural network attributes.

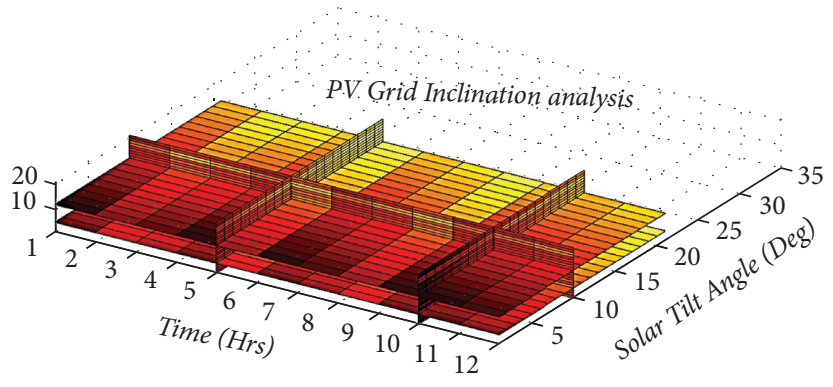

Figure 7: PV grid inclination analysis.

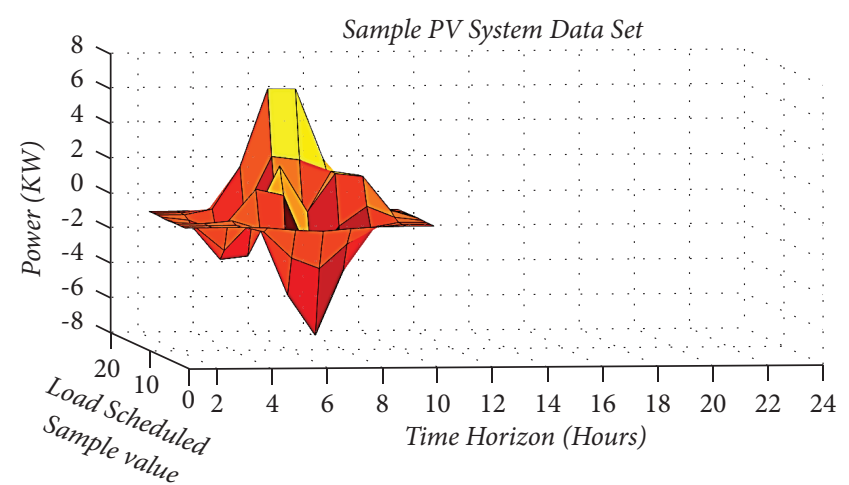

Figure 8: Sample PV dataset.

power to the load, energy storage system is incorporated. Since the insolation is varied in nature based on time horizon, the designed model is considered to fall under nonlinear system analysis and outstrips using gradient descent and conjugate gradient methods. Based on sample PV system data, 10 neurons were initiated in the hidden layer and later the number of neurons in the hidden layer is increased depending upon the load schedule. The interpolation of the PV system towards the forecasted load schedule is shown in Figure 9. Peak power availability and its utilization towards EV and stationary system load demand are shown in Figure 9.

The sample dataset shown in Figure 8 is utilized by the prediction model. The sample dataset is subclassified as training set, validating set, and testing set, in which $70 \%$ of

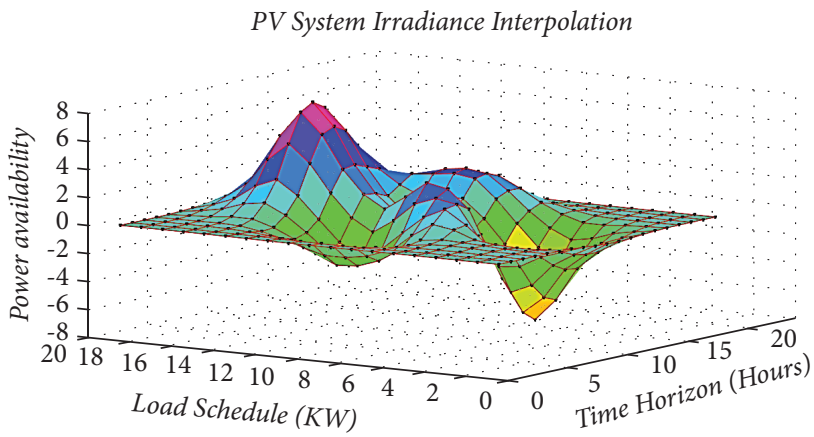

FIgURE 9: PV system interpolation towards load schedule.

data are considered as training set and remaining 30\% are considered as validating and testing sets.

The output shown in Figure 10 shows SOC of V2G service that regulates the frequency on the interconnected grid system on 24-hour basic. The V2G service has two main functions which control the charging on to the connected battery and use the available power to regulate the grid.

Figure 10 shows the battery SOC status at 5 different profiles.

Profile \#1: Figure 10(a) shows the status of battery charging status. During this stage, the battery gets charged to a maximum value of $95 \%$. Most of the time, the battery is connected to the system resources and gets charged to a maximum value.

Profile \#2: as shown in Figure 10(b), the battery is in the charging state but is no longer used to supply load. In 


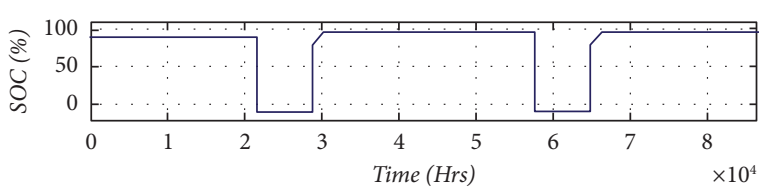

(a)

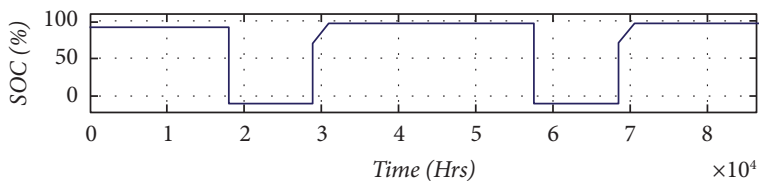

(c)

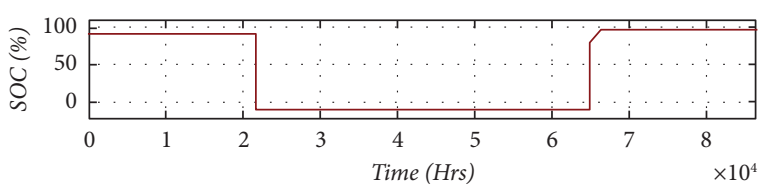

(b)

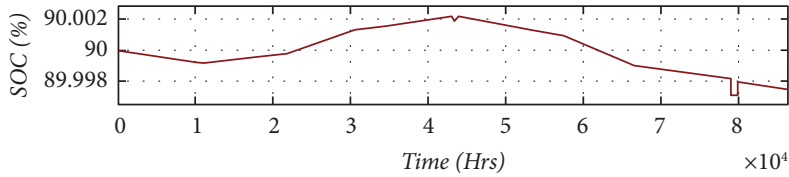

(d)

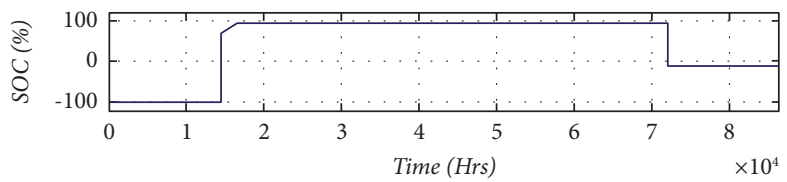

(e)

FIGURE 10: Battery SOC status at various profiles.

this condition, the PV system charges the battery but does not supply power to load. From $t=2$ to $t=6.5$, the battery power is drained and the SOC value is approximately $0 \%$. Again from $t=6.5$, the battery starts to charge from the source.

Profile \#3: as shown in Figure $10(\mathrm{c})$, the battery is connected, but there is no possibility to charge.

Profile \#4: as shown in Figure 10(d), the battery is in the ideal state. During this condition, the range of SOC varies between 89.998 and $90.002 \%$.

Profile \#5: as shown in Figure 10(e), during night time, the solar PV system does not deliver any power, and at that moment, the battery connected in the system supplies power to the charging EV station.

\section{Conclusion}

A centralized energy management strategy is proposed in this paper for EV system charging and discharging strategy, so as to reduce the tariff rate. The prediction models were investigated using SSNN to predict the availability of solar power at various time horizons. Based on the availability of power, the load forecasting schedule is proposed for EV charging/discharging and the stationary battery storage system. The SSNN-based EMS strategy is designed based on weight adjustment, where in the simulation process, $70 \%$ of data are considered as training set and the remaining 30\% are considered as validating and testing sets. The weight adjustment finds the exact EV availability status at different atmospheric conditions as $\mathrm{EV}$ Status $=4 \& 2$ for $\mathrm{EV}$ (availability-1) $\mathbb{E} \mathbb{V}_{t-1}=1$ and $\mathrm{EV}$ status $=3$ \& 2 for $\mathrm{EV}$ (availability-1) $\mathbb{E V} \mathbb{t}_{t-1}=0$. The status of power drawn is given in equation (5) when $\alpha=1$ and $\beta=0$; under this condition, the power drawn from the grid is positive. Energy is sold from the grid, when $\alpha=0$ and $\beta=1$, and under this condition, the power drawn from the grid is negative. The modeling and simulation test results indicate that the designed CEMS is more effective in terms of reducing the tariff rate and load sharing. The relevance of the proposed work provides better V2G service and supplies high-quality power to the scheduled EV load and stationary load. As future enhancement, atmospheric disaster can be considered and big data analysis can be incorporated to further enhance the system security with effective load sharing in the grid connected smart system. The role of big data analysis in optimization techniques can be applied to reduce the uncertainty caused by the connected renewable energy resources. The power generation by nondispatchable energy conversion technologies is highly variable and unpredictable; that leads to the technology of developing and planning for an optimized operation tool in order to maximize the economy with sustained performance.

\section{Nomenclature}

EMS: $\quad$ Energy management strategy

CCS: $\quad$ Centralized control strategy

DCS: Decentralized control strategy

DBM: Degradation battery model

MILP: $\quad$ Mixed-integer linear programming

SSNN: $\quad$ Smart superficial neural network

PDF: Probability distribution function

SOC: $\quad$ State of charge

SOH: $\quad$ State of health

PCC: $\quad$ Point of common coupling

ANN: Artificial neural network

LMA: Levenberg-Marquardt algorithm

V2G: Vehicle-to-grid

RTS: $\quad$ Real-time system

RES: $\quad$ Renewable energy source

HEMS: Hybrid energy management system

MAHEMS: Multiagent hybrid energy management system

LVDS: Low-voltage distribution system. 


\section{Data Availability}

The data used to support the findings of this study are available from the corresponding author upon request.

\section{Conflicts of Interest}

The authors declare that they have no conflicts of interest.

\section{References}

[1] S. Gangatharan, M. Rengasamy, R. M. Elavarasan, N. Das, E. Hossain, and V. M. Sundaram, "A novel battery supported energy management system for the effective handling of feeble power in hybrid microgrid environment," IEEE Access, vol. 8, pp. 217391-217415, 2020.

[2] J. Nunez Forestieri and M. Farasat, "Integrative sizing/realtime energy management of a hybrid supercapacitor/undersea energy storage system for grid integration of wave energy conversion systems," IEEE Journal of Emerging and Selected Topics in Power Electronics, vol. 8, no. 4, pp. 3798-3810, Dec. 2020.

[3] H. Novak, V. Vasak, and M. Vašak, "Hierarchical model predictive control for coordinated electric railway traction system energy management," IEEE Transactions on Intelligent Transportation Systems, vol. 20, no. 7, pp. 2715-2727, July 2019.

[4] R. H. Byrne, T. A. Nguyen, D. A. Copp, B. R. Chalamala, and I. Gyuk, "Energy management and optimization methods for grid energy storage systems," IEEE Access, vol. 6, pp. 13231-13260, 2018.

[5] B. Zhao, X. Wang, D. Lin et al., "Energy management of multiple microgrids based on a system of systems architecture," IEEE Transactions on Power Systems, vol. 33, no. 6, pp. 6410-6421, 2018.

[6] M. T. Lawder, B. Suthar, P. W. C. Northrop et al., "Battery energy storage system (BESS) and battery management system (BMS) for grid-scale Applications," Proceedings of the IEEE, vol. 102, no. 6, pp. 1014-1030, June 2014.

[7] J. Han, C.-s. Choi, W.-k. Park, I. Lee, and S.-h. Kim, "Smart home energy management system including renewable energy based on ZigBee and PLC," IEEE Transactions on Consumer Electronics, vol. 60, no. 2, pp. 198-202, May 2014.

[8] T. Kovaltchouk, A. Blavette, J. Aubry, H. B. Ahmed, and B. Multon, "Comparison between centralized and decentralized storage energy management for direct wave energy converter farm," IEEE Transactions on Energy Conversion, vol. 31, no. 3, pp. 1051-1058, Sept. 2016.

[9] S. Aminzadeh and A. Ejlali, "A comparative study of systemlevel energy management methods for fault-tolerant hard real-time systems," IEEE Transactions on Computers, vol. 60, no. 9, pp. 1288-1299, 2011.

[10] M. Mao, P. Jin, N. D. Hatziargyriou, and L. Chang, "Multiagent-based hybrid energy management system for microgrids," IEEE Transactions on Sustainable Energy, vol. 5, no. 3, p. 1, July 2014.

[11] C. Molitor, S. Gross, J. Zeitz, and A. Monti, "MESCOS-A m system c for city district energy systems," IEEE Transactions on Industrial Informatics, vol. 10, no. 4, pp. 2247-2256, 2014.

[12] G. K. Venayagamoorthy, R. K. Sharma, P. K. Gautam, and A. Ahmadi, "Dynamic energy management system for a smart microgrid," IEEE Transactions on Neural Networks and Learning Systems, vol. 27, no. 8, pp. 1643-1656, Aug. 2016.
[13] N. Nishikawa, M. Nakano, and M. Kitsuregawa, "Application sensitive energy management framework for storage systems," IEEE Transactions on Knowledge and Data Engineering, vol. 27, no. 9, pp. 2335-2348, 1 Sept. 2015.

[14] C. Zheng, W. Li, and Q. Liang, "An energy management strategy of hybrid energy storage systems for electric vehicle applications," IEEE Transactions on Sustainable Energy, vol. 9, no. 4 , pp. $1880-1888$, Oct. 2018.

[15] A. M. O. Haruni, M. Negnevitsky, M. E. Haque, and A. Gargoom, "A novel operation and control strategy for a standalone hybrid renewable power system," IEEE Transactions on Sustainable Energy, vol. 4, no. 2, pp. 402-413, April 2013. 